\title{
"Hábitos de estudio y rendimiento académico de los estudiantes de la facultad de educación de la universidad nacional José Faustino Sánchez Carrión - sede Huaral durante el año académico $2015 "$
}

\section{"Habits of study and academic performance of the students of the faculty of education of the national university José Faustino Sánchez Carrión - Huaral headquarters during the academic year $2015^{\prime \prime}$}

Lic. Nicolás Huertas Pizarro

Escuela Profesional de Educación / Universidad Nacional de Educación Enrique Guzmán y Valle

\section{RESUMEN}

La investigación científica que se ha realizado es básica, correlacional causal no experimental, los factores de estudio son dos: hábitos de estudio y rendimiento académico en los estudiantes de la Facultad de Educación de la Universidad Nacional José Faustino Sánchez Carrión.

El diseño de la investigación es transeccional, correlacional, bivariada, transversal. Para la recolección de datos se confeccionó un cuestionario estructurado. En cuanto al rendimiento académico, se utilizó la técnica de análisis documental trabajando con las calificaciones de los estudiantes, se aplicó a 100 estudiantes.

El instrumento fue adaptado del inventario de hábitos de estudio de Luis Alberto Vicuña Peri cumpliendo con las cualidades de validez y confiabilidad. El estudio plantea la siguiente hipótesis de investigación: existe relación significativa entre los hábitos de estudio y el rendimiento académico.

Los resultados obtenidos permiten primero evidenciar que existe una correlación alta y significativa entre los hábitos de estudio y el rendimiento académico de los estudiantes de la Facultad de Educación de la Universidad Nacional José Faustino Sánchez Carrión -sede Huaral- durante el año académico 2015, con un Rho de Spearman $=.749 \quad$ y $p<.05$.

Palabras clave: Hábitos de estudio, rendimiento, estudiantes. 


\begin{abstract}
The scientific research that has been carried out is basic, non experimental causal correlational, the study factors are two: study habits and academic performance in the students of the faculty of education of the national university José Faustino Sánchez Carrión.

The research design is transectional, correlational, bivariate, cross-sectional. For data collection, a structured questionnaire was prepared. As for academic performance, the documentary analysis technique was used, working with the students' grades, applied to 100 students.

The instrument was adapted from the inventory of study habits of Luis Alberto Vicuña Peri fulfilling the qualities of validity and reliability. The study raises the following research hypothesis there is a significant relationship between study habits and academic performance.

The obtained results allow first to show that there is a high and significant correlation between study habits and the academic performance of the students of the Faculty of Education of the National University José Faustino Sánchez Carrión - Huaral campus - during the academic year 2015, with a Spearman's Rho $=.749$ and $p<.05$.
\end{abstract}

Key words: Study habits, performance, students.

\section{Introducción}

La observación empírica ha hecho evidente en repetidas ocasiones la dificultad de muchos estudiantes de la Facultad de Educación de la Universidad Nacional José Faustino Sánchez Carrión, para organizar sus actividades académicas. Muchas señales muestran que la situación problemática de presenta de modo altamente frecuente: El incumplimiento en la presentación de trabajos, distracción durante las clases, respuestas inadecuadas a preguntas formuladas durante las sesiones y otras señales que permiten conjeturar deficiencias notables que podrían tener como causa una adquisición de inadecuados hábitos de estudio. Los resultados obtenidos en las evaluaciones reflejan en muchos casos problemas de rendimiento académico que ocasionan retrasos en la culminación de la carrera cuando no el abandono de los estudios.

Los factores que determinan el rendimiento académico son muchos y muy diversos, pudiéndose según la lógica propuesta por Carlos Alberto Seguín, clasificarlos como factores predisponentes, determinantes y desencadenantes. A pesar de la complejidad del panorama, se ha optado por indagar la relación existente entre los hábitos de estudio y el rendimiento académico de los alumnos objeto del presente estudio por considerar que ambos son aspectos más altamente críticos y sobre los cuales existen evidencias científicas de relación causal. La problemática que ocupa esta tesis ha sido frecuentemente abordada en investigaciones tanto nacionales como internacionales, encontrándose que la situación es igualmente crítica en todo el ámbito del el sistema universitario peruano y el sistema educativo en general. No obstante la abundante cantidad de estudios sobre el rendimiento académico y los hábitos de estudio, se ha considerado que sigue siendo necesario el abordaje del tema mientras no se hayan implementado medidas para su afrontamiento. Los resultados del presente estudio podrán ser de utilidad no sólo para la Facultad de Educación sino para la universidad toda, de manera que a la luz de las conclusiones ofrecidas se podrá tomar medidas que permitan mejorar la calidad del servicio educativo y producir con ello un mejor producto en la formación de los futuros profesionales.

Se ha encontrado los siguientes antecedentes de estudio: Cartagena (2008), realizó la investigación cuyo propósito fue de comparar los puntajes de auto eficiencia en el rendimiento académico como los aspectos de los hábitos de estudio referidos a técnicas, organización, tiempo, distractibilidad en los alumnos de $1^{\text {o }}$, $2^{\circ}, 5^{\circ}$ de secundaria del Colegio Técnico Parroquial Peruano Chino "San Francisco de Asís", ubicado en el distrito de Ate VitarteHuaycán de la UGEL 06de Lima, con alto y bajo rendimiento académico. Los resultados demuestran que existen diferencia significativa entre los niveles de los hábitos de estudio de los alumnos de $1^{\circ}, 2^{\circ}$ y $5^{\circ}$ de secundaria, con alto y bajo rendimiento.

Ortega (2012) en la investigación sobre los hábitos de estudio y rendimiento académico en estudiantes de segundo de secundaria de una institución educativa del Callao, demuestra que existe una relación directa alta entre los niveles 
de hábitos de estudio y los niveles del rendimiento académico; además concluye que los estudiantes carecen de métodos de estudio, no tienen una hora fija de estudio y el tiempo de dedicación para el estudio es variado dependiendo de la complejidad de la tarea.

Fermín (2003) en Venezuela en la investigación realizada sobre relación entre hábitos de estudio y rendimiento estudiantil, en los alumnos del CEAPUCV durante el año escolar 2003- 2004, concluye que a mayor presencia de hábitos de estudio, se obtendrá un mejor rendimiento académico y a menos presencia menor será el mismo.

Carlos Loret de Mola (2011) en la investigación realizada sobre "relación existente entre los estilos y estrategias de aprendizaje y el rendimiento académico en los estudiantes de la Universidad Peruana "Los Andes" de la Facultad de Educación y Ciencias Humanas, llegó a concluir que el estilo de aprendizaje predominante es el reflexivo porque 73 estudiantes obtuvieron el nivel alto, enseguida está el estilo activo, también el estilo teórico y por último se ubica el estilo pragmático con 50 estudiantes con el $37 \%$ del total de la muestra; demostrando que los estudiantes tienen una diversidad de estilos de aprendizaje al momento de desarrollar sus actividades cognitivas.

Luque Mario (2006) realizó una investigación cuyo propósito fue determinar la influencia de los hábitos de estudio en el rendimiento académico del área de matemática de los alumnos de la I.E.S de Cabana-2006, Perú, logrando concluir que los hábitos de estudio influyen en el rendimiento académico de los estudiantes del cuarto y quinto grado de educación secundaria en el área de matemática.

La situación descrita permite formular la siguiente interrogante: ¿Cuál es el la relación entre los hábitos de estudio y el rendimiento académico de los estudiantes de la Facultad de Educación de la Universidad Nacional José Faustino Sánchez Carrión -sede Huaral- durante el año académico 2015?

Los objetivos del estudio fueron: (1) Determinar la relación entre las formas de estudio y el rendimiento académico de los estudiantes de la Facultad de Educación de la Universidad Nacional José Faustino Sánchez Carrión -sede Huaral- durante el año académico 2015. (2) Identificar la relación entre la resolución de tareas y el rendimiento académico de los estudiantes de la Facultad de Educación de la Universidad Nacional José Faustino Sánchez Carrión -sede Huaral- durante el año académico 2015. (3) Establecer la relación entre la preparación para los exámenes y el rendimiento académico de los estudiantes de la Facultad de Educación de la Universidad Nacional José Faustino Sánchez Carrión -sede Huaral- durante el año académico 2015. (4) Establecer la relación entre la forma de escuchar la clase y el rendimiento académico de los estudiantes de la Facultad de Educación de la Universidad Nacional José Faustino Sánchez Carrión -sede Huaral- durante el año académico 2015. La hipótesis se formulada fue: Existe relación entre los hábitos de estudio y el rendimiento académico de los estudiantes de la Facultad de Educación de la Universidad Nacional José Faustino Sánchez Carrión -sede Huaral- durante el año académico 2015.

\section{Material y métodos}

\section{Objeto del estudio}

La unidad de análisis del presente trabajo es un alumno de la Facultad de Educación de la Universidad Nacional José Faustino Sánchez Carrión -sede Huaral.

\section{Población y muestra}

La población está constituida 60 estudiantes de la Facultad de Educación de la Universidad Nacional José Faustino Sánchez Carrión - sede Huaral-durante el año académico 2015. Considerando la posibilidad de acceder al total de estudiantes no se recurrió a determinar un tamaño muestral menor que la población, siendo entonces la población igual a la muestra.

\section{Instrumentos de recolección de datos}

Cuestionario de evaluación de la variable hábitos de estudio. Autor: Luis Alberto Vicuña Peri. Adaptado por: Lic. Nicolás Huertas Pizarro.

\section{Método}

\section{Técnicas}

Para el acopio de datos referentes a los hábitos de estudio, se aplicó la técnica de encuesta. Para el estudio del rendimiento académico, se recurrió al análisis documental.

\section{Diseño de estudio}

Se ha optado por el diseño correlacional, determinándose el valor de la correlación mediante el coeficiente de correlación de Speraman.

\section{Procedimiento:}

- Se coordinó con La Institución Educativa y se obtiene los permisos para la evaluación de los estudiantes. 
- Se aplicó los instrumentos de acopio de datos.

- Se tabuló, analizó y discutió los datos.
- Se redactó las conclusiones y sugerencias en base a la discusión de resultados

- Se comunicaron las conclusiones del trabajo de investigación.

\section{Resultados}

Porcentaje de la variable Hábitos de Estudio

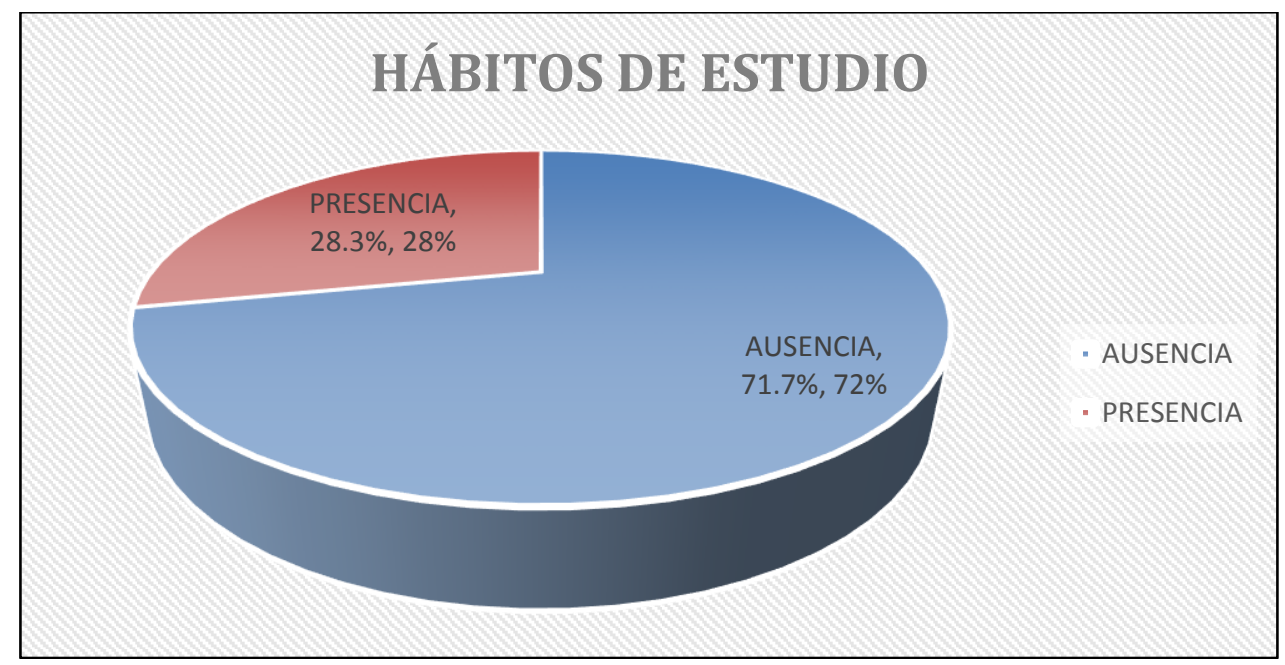

Figura $N^{\boldsymbol{o}}$ 1. Se encontró que el 71,75\% (43) de los estudiantes encuestados se encuentran en el nivel de ausencia con respecto a la variable hábitos de estudio, mientras que el 28,3\% (17) se ubican en el nivel de presencia.

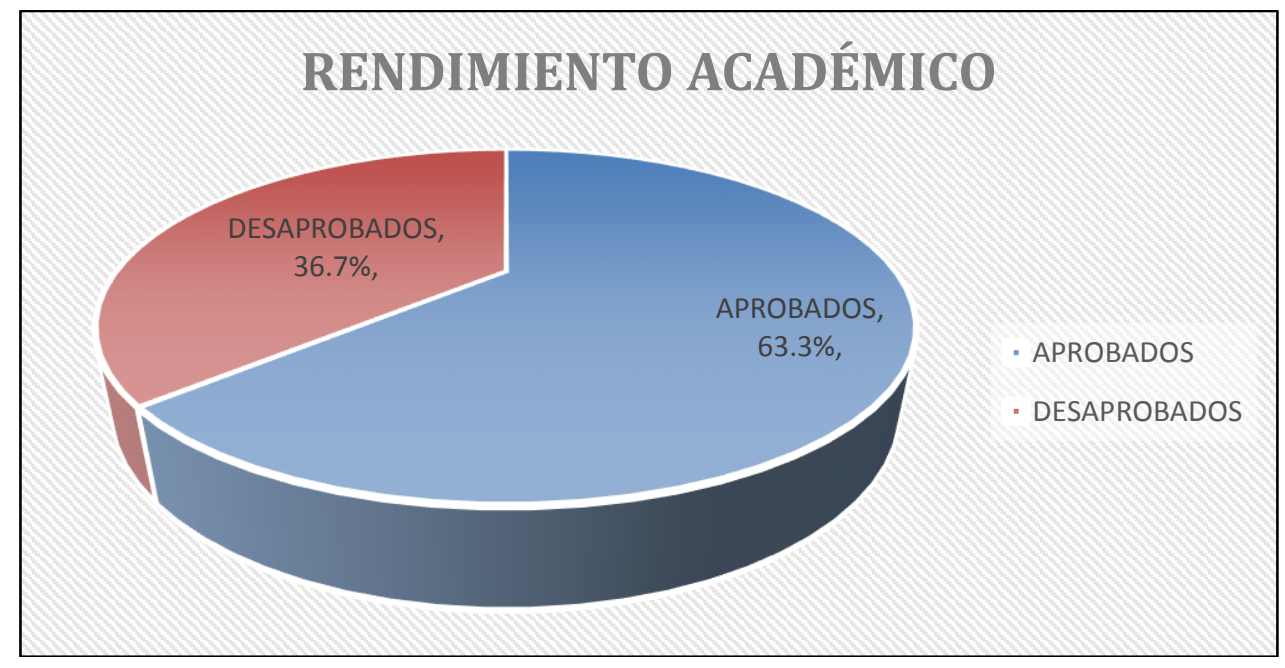

Figura $N^{\circ}$ 2. El 63,3\% se encuentran desaprobados; 22 encuestados que equivale al $36,7 \%$ se encuentran aprobados los estudiantes de la Facultad de Educación de la Universidad Nacional José Faustino Sánchez Carrión-sede Huaral-durante el año académico 2015. 
Tabla $N^{o} 1$. Correlación entre Hábitos de Estudio y Rendimiento Académico

\begin{tabular}{|c|c|c|c|c|}
\hline & & & $\begin{array}{l}\text { Hábitos de } \\
\text { estudio }\end{array}$ & $\begin{array}{l}\text { Rendimiento } \\
\text { académico }\end{array}$ \\
\hline \multirow{6}{*}{$\begin{array}{l}\text { Rho de } \\
\text { Spearman }\end{array}$} & \multirow[t]{3}{*}{ Hábitos de estudio } & Coeficiente de correlación & 1,000 &, $749^{* *}$ \\
\hline & & Sig. (bilateral) & . & 000, \\
\hline & & $\mathrm{N}$ & 60 & 60 \\
\hline & \multirow[t]{3}{*}{$\begin{array}{l}\text { Rendimiento } \\
\text { académico }\end{array}$} & Coeficiente de correlación &, $749^{* *}$ & 1,000 \\
\hline & & Sig. (bilateral) & 000 & \\
\hline & & $\mathrm{N}$ & 60 & 60 \\
\hline
\end{tabular}

Al aplicar la formula Rho de Spearman margen de error al 95\% el coeficiente de correlación es $\mathrm{r}=, 749 * *$ y el $p_{\text {_ }}$ valor es de 0,000 , por lo tanto se establece un nivel correlación buena, existe una relación significativa por lo tanto se rechaza la hipótesis Nula y se acepta la alterna Existe relación entre los hábitos de estudio y el rendimiento académico de los estudiantes de la Facultad de Educación de la Universidad Nacional José Faustino Sánchez Carrión -sede Huaral- durante el año académico 2015.

Tabla $\mathbf{N}^{\circ}$ 2. Correlación entre la dimensión formas de estudio y el rendimiento académico

\begin{tabular}{|c|c|c|c|c|}
\hline & & & $\begin{array}{c}\text { Formas de } \\
\text { estudio }\end{array}$ & $\begin{array}{l}\text { Rendimiento } \\
\text { académico }\end{array}$ \\
\hline \multirow{6}{*}{$\begin{array}{l}\text { Rho de } \\
\text { Spearman }\end{array}$} & Formas de estudio & Coeficiente de correlación & 1,000 &, $786^{* *}$ \\
\hline & & Sig. (bilateral) & . &, 000 \\
\hline & & $\mathrm{N}$ & 60 & 60 \\
\hline & $\begin{array}{l}\text { Rendimiento } \\
\text { académico }\end{array}$ & Coeficiente de correlación &, $786^{* *}$ & 1,000 \\
\hline & & Sig. (bilateral) &, 000 & . \\
\hline & & $\mathrm{N}$ & 60 & 60 \\
\hline
\end{tabular}

Al aplicar la formula Rho de Spearman margen de error al 95\% el coeficiente de correlación es $\mathrm{r}=, 786^{* *}$ y el p_ valor es de 0,000 , por lo tanto se establece un nivel correlación buena, existe una relación significativa entre las formas de estudio y el rendimiento académico.

Tabla No 3. Correlaciones entre la dimensión Resolución de Tareas y el Rendimiento Académico

\begin{tabular}{|c|c|c|c|c|}
\hline & & & $\begin{array}{l}\text { Resolución de } \\
\text { tareas }\end{array}$ & $\begin{array}{l}\text { Rendimiento } \\
\text { académico }\end{array}$ \\
\hline \multirow{5}{*}{$\begin{array}{l}\text { Rho de } \\
\text { Spearman }\end{array}$} & \multirow{3}{*}{$\begin{array}{l}\text { Resolución de } \\
\text { tareas }\end{array}$} & Coeficiente de correlación & 1,000 &, $705^{* *}$ \\
\hline & & Sig. (bilateral) & . & ,000 \\
\hline & & $\mathrm{N}$ & 60 & 60 \\
\hline & \multirow{2}{*}{$\begin{array}{l}\text { Rendimiento } \\
\text { académico }\end{array}$} & Coeficiente de correlación &, $705^{* *}$ & 1,000 \\
\hline & & Sig. (bilateral) & ,000 & . \\
\hline \multicolumn{3}{|c|}{ **. La correlación es significativa al nivel 0,01 (bilateral). } & 60 & 60 \\
\hline
\end{tabular}


Con margen de error al 95\% el coeficiente de correlación es $\mathrm{r}=0,705$ y el p_ valor es de 0,000, por lo tanto se establece un nivel correlación buena. Existe relación entre la resolución de tareas y el rendimiento académico.

Tabla $N^{\circ}$ 4. Correlaciones entre la dimensión Preparación para los Exámenes y el Rendimiento Académico

\begin{tabular}{|c|c|c|c|c|}
\hline & & & $\begin{array}{c}\text { Preparación para los } \\
\text { exámenes }\end{array}$ & $\begin{array}{l}\text { Rendimiento } \\
\text { académico }\end{array}$ \\
\hline \multirow[t]{6}{*}{$\begin{array}{l}\text { Rho de } \\
\text { Spearman }\end{array}$} & $\begin{array}{l}\text { Preparación para } \\
\text { los exámenes }\end{array}$ & $\begin{array}{l}\text { Coeficiente de } \\
\text { correlación }\end{array}$ & 1,000 &, $740^{* * *}$ \\
\hline & & Sig. (bilateral) & . & ,000 \\
\hline & & $\mathrm{N}$ & 60 & 60 \\
\hline & $\begin{array}{l}\text { Rendimiento } \\
\text { académico }\end{array}$ & $\begin{array}{l}\text { Coeficiente de } \\
\text { correlación }\end{array}$ &, $740^{* * *}$ & 1,000 \\
\hline & & Sig. (bilateral) & ,000 & . \\
\hline & & $\mathrm{N}$ & 60 & 60 \\
\hline
\end{tabular}

Al aplicar la formula Rho de Spearman margen de error al 95\% el coeficiente de correlación es $\mathrm{r}=0,740$ y el p_ valor es de 0,000 , por lo tanto se establece un nivel correlación buena. Existe relación entre la preparación para los exámenes y el rendimiento académico.

Tabla $\mathbf{N}^{\mathbf{0}}$ 5. Correlaciones entre la dimensión Formas de Escuchar la Clase y el Rendimiento Académico

\begin{tabular}{|c|c|c|c|c|}
\hline & & & $\begin{array}{l}\text { Formas de escuchar la } \\
\text { clase }\end{array}$ & $\begin{array}{l}\text { Rendimiento } \\
\text { académico }\end{array}$ \\
\hline \multirow[t]{6}{*}{$\begin{array}{l}\text { Rho de } \\
\text { Spearman }\end{array}$} & \multirow[t]{3}{*}{$\begin{array}{l}\text { Formas de escuchar } \\
\text { la clase }\end{array}$} & $\begin{array}{l}\text { Coeficiente de } \\
\text { correlación }\end{array}$ & 1,000 &, $736^{* *}$ \\
\hline & & Sig. (bilateral) & &, 000 \\
\hline & & $\mathrm{N}$ & 60 & 60 \\
\hline & \multirow[t]{3}{*}{$\begin{array}{l}\text { Rendimiento } \\
\text { académico }\end{array}$} & $\begin{array}{l}\text { Coeficiente de } \\
\text { correlación }\end{array}$ &, $736^{* *}$ & 1,000 \\
\hline & & Sig. (bilateral) & ,000 & . \\
\hline & & $\mathrm{N}$ & 60 & 60 \\
\hline
\end{tabular}

Al aplicar la formula Rho de Spearman margen de error al 95\% el coeficiente de correlación es $\mathrm{r}=0,736$ y el p_ valor es de 0,000 , por lo tanto se establece un nivel correlación buena. Existe relación entre la forma de escuchar la clase y el rendimiento académico. 
Tabla $\mathbf{N}^{0}$ 6. Correlaciones entre la dimensión Acompañamiento al Estudio y el Rendimiento Académico

\begin{tabular}{|c|c|c|c|c|}
\hline & & & $\begin{array}{c}\text { Acompañamiento al } \\
\text { estudio }\end{array}$ & $\begin{array}{l}\text { Rendimiento } \\
\text { académico }\end{array}$ \\
\hline \multirow[t]{6}{*}{$\begin{array}{l}\text { Rho de } \\
\text { Spearman }\end{array}$} & $\begin{array}{l}\text { Acompañamiento al } \\
\text { estudio }\end{array}$ & $\begin{array}{l}\text { Coeficiente de } \\
\text { correlación }\end{array}$ & 1,000 &, $596^{* *}$ \\
\hline & & Sig. (bilateral) & . &, 000 \\
\hline & & $\mathrm{N}$ & 60 & 60 \\
\hline & $\begin{array}{l}\text { Rendimiento } \\
\text { académico }\end{array}$ & $\begin{array}{l}\text { Coeficiente de } \\
\text { correlación }\end{array}$ &, $596^{* *}$ & 1,000 \\
\hline & & Sig. (bilateral) &, 000 & . \\
\hline & & $\mathrm{N}$ & 60 & 60 \\
\hline
\end{tabular}

Con un margen de error al 95\% el coeficiente de correlación es $r=0,596$ y el p_ valor es de 0,000, por lo tanto se establece un nivel correlación buena. Existe relación entre la dimensión acompañamiento al estudio y el rendimiento académico de los estudiantes de la Facultad de Educación de la Universidad Nacional José Faustino Sánchez Carrión -sede Huaral- durante el año académico 2015.

\section{Discusión de los resultados}

La presente investigación demostró que existe correlación significativa entre las variables hábitos de estudio y el rendimiento académico de los estudiantes de la Facultad de Educación de la Universidad Nacional José Faustino Sánchez Carrión -sede Huaral- durante el año académico 2015, con Rho de Spearman= 749, al respecto la investigación que realizo Vildoso (2003) llega a la conclusión que existe correlación significativa entre los hábitos de estudio, la autoestima y el rendimiento académico de los alumnos del segundo, tercero y cuarto año, los porcentajes obtenidos muestran que existe un considerable grupo de estudiantes que presentan un nivel bajo de hábitos de estudio. Luque (2006) realizo una investigación en la que llego a la conclusión que existe influencia de los hábitos de estudio en el rendimiento académico.

Así mismo, también se encontró que existe correlación entre la dimensión formas de estudio y el rendimiento académico al encontrarse el valor de Rho de Spearman=,786 las formas de estudio están referidas a cómo debe estudiar el alumno para obtener un mejor rendimiento, en la investigación que realizo Argentina (2013) concluye que la práctica de buenos hábitos de estudio influye en el rendimiento académico, de los buenos hábitos de estudio depende el éxito que se obtengan durante el recorrido estudiantil hasta la vida profesional.
En cuanto a la segunda dimensión se encontró que existe una correlación entre la dimensión resolución de tareas y el rendimiento académico, con Rho de Spearman= 705, la investigación de Ortega (2012) titulada "Hábitos de estudio y rendimiento académico en estudiantes de segundo de secundaria de una institución educativa del callao, en la que llega a la conclusión que los estudiantes carecen de métodos de estudio, no tienen una hora fija de estudio y el tiempo de dedicación para el estudio es variado dependiendo de la complejidad de la tarea.

Respecto a la tercera dimensión se encontró que existe una correlación entre la dimensión preparación para los exámenes y el rendimiento académico con Rho de Spearman=, 740, al respecto la investigación de Castaño, Giraldo, Peralta y Ramírez (2006) concluye que existen diferencias significativas de los factores resilientes entre uno de estos factores se encuentra la habilidad de resolver problemas como los exámenes que se plantean para que los estudiantes con alto rendimiento académico

En cuanto a la cuarta dimensión se observa que existe una correlación entre la dimensión formas de escuchar la clase y el rendimiento académico con Rho de Spearman= ,736 en la investigación de Fermín (2003) concluye que hay factores que resultaron muy positivos como lo son: Ambiente de Estudio, Habilidades de Lectura y la Motivación para el Estudio; pero hay otros que requieren ser fortalecidos como: Métodos de Estudio entre los cuales se debe tener en cuenta 
como el estudiante escucha las clases y Planificación del Estudio

Finalmente la quinta dimensión se obtuvo que existe una correlación entre la dimensión acompañamiento al estudio y el rendimiento académico con Rho de Spearman=,596 la investigación que realizo Esquerra y Ospina (2015) concluye que existe relación significativa entre los estilos de aprendizaje y el rendimiento académico, especialmente en estudiantes que muestran el estilo activo y reflexivo.

\section{Conclusiones}

- Se evidencio existe una correlación alta y significativa entre los hábitos de estudio y el rendimiento académico de los estudiantes de la Facultad de Educación de la Universidad Nacional José Faustino Sánchez Carrión sede Huaral- durante el año académico 2015, con un Rho de Spearman $=.749$ y $\mathrm{p}<.05$.

- En cuanto a la hipótesis especifica primera se encontró una correlación alta y significativa entre las formas de estudio y el rendimiento académico de los estudiantes de la Facultad de Educación de la Universidad Nacional José Faustino Sánchez Carrión sede Huaral- durante el año académico 2015, con un Rho de Spearman $=.786$ y $\mathrm{p}<.05$.

- Con respecto a la hipótesis especifica dos se determinó una correlación alta y significativa entre resolución de tareas y el rendimiento académico de los estudiantes de la Facultad de Educación de la Universidad Nacional José Faustino Sánchez Carrión sede Huaral- durante el año académico 2015, con un Rho de Spearman $=.705$ y $\mathrm{p}<.05$.

- Se encontró una correlación de $\mathrm{r}=0.740 \mathrm{y}$ $\mathrm{p}<.05$ por lo que se rechaza la hipótesis nula y se acepta la hipótesis alterna, afirmándose existe relación entre la preparación para los exámenes y el rendimiento académico de los estudiantes de la Facultad de Educación de la Universidad Nacional José Faustino Sánchez Carrión sede Huaral- durante el año académico 2015.

- Se encontró una correlación de $\mathrm{r}=0.736$ por lo que se rechaza la hipótesis nula y se acepta la hipótesis alterna, afirmándose existe relación significativa entre las formas de escuchar y el rendimiento académico de los estudiantes de la Facultad de Educación de la Universidad Nacional José Faustino Sánchez Carrión -sede Huaral- durante el año académico 2015.

- Se encontró una correlación de $\mathrm{r}=0.596$ por lo que se rechaza la hipótesis nula y se acepta la hipótesis alterna, afirmándose existe relación significativa entre el nivel de acompañamiento al estudio y el rendimiento académico de los estudiantes de la Facultad de Educación de la Universidad Nacional José Faustino Sánchez Carrión -sede Huaral- durante el año académico 2015.

\section{Referencias bibliográficas}

[1] Alcalay, L. y Antonijevic, N. (1987). Variables afectivas. Revista de Educación (México), 1 (44), 29-32. Recuperado de http://www.redalyc.org/pdf/132/13206011. pdf

[2] Almaguer, T. (1 998). El desarrollo del alumno: características y estilos de aprendizaje. México: Trillas.

[3] Argentina (2013) Relación entre hábitos de estudio y rendimiento académico en estudiantes de primero básico. Universidad Rafael Landivar

[4] Benítez, M; Gimenez, M. y Osicka, R. (2000). Las asignaturas pendientes y el rendimiento académico: ¿existe alguna relación? En red. Recuperado en: http//fai.unne.edu.ar/links/LAS\%2...20EL $\%$ 20rendimiento\%20academico.htm

[5] Benítez, M; Gimenez, M. y Osicka, R. (2000). Las asignaturas pendientes y el rendimiento académico: ¿existe alguna relación? Recuperado de http://www.unne.edu.ar/unnevieja/Web/cyt /cyt/humanidades/h-009.pdf

[6] Cantú (2004) en su tesis "Estilo de aprendizaje y su relación con el desempeño académico de los estudiantes de arquitectura de la Universidad Autónoma de México".

[7] Cartagena, M. (2008) Relación entre auto eficiencia y el rendimiento escolar y los hábitos de estudio en alumnos de secundaria. Lima

[8] Cascón, I. (2000). Análisis de las calificaciones escolares como criterio de rendimiento académico. Recuperado de: http://www3.usal.es./inico/investigacion/jo rnadas/jornada2/comunc/cl7.html

[9] Castejón, J. (2015). Aprendizaje y rendimiento académico. Alicante - España:

[10] Cerda, H. (2003). La nueva evaluación educativa. Desempeños, logros, competencias y estándares. Colombia: Cooperativa Editorial Magisterio

[11] Club Universitario Covington, M. (1984). The motive for self-worth. En R. Ames y C. Ames (Eds.). Research on Motivation in 
Education. Student Motivation. Vol.I. New York: Academic Press.

[12] De Giraldo, L.; Mera, R. (2000). Clima social escolar: percepción del estudiante. Recuperado en: http: //colombiamedica.univalle.edu.co/Vol31N o1/clima.html

[13] Díaz, A. (2012) Relación entre los estilos de aprendizaje y el rendimiento académico de las estudiantes de la Escuela Profesional de Enfermería de la Facultad de Ciencias de la Salud de la Universidad Nacional del Callao. Tesis para obtener el grado de Magister. Universidad Nacional del Callao. Perú

[14] Fermín, I. (2003). Relación entre hábitos de estudio y rendimiento estudiantil. Recuperado de http://www.monografias.com/trabajos26/h abitos-estudio/habitosestudio2.shtml\#ixzz3fuiQg39y

[15] Garbanzo, G. (2007). Factores asociados al rendimiento académico en estudiantes universitarios, una reflexión desde la calidad de la educación superior pública. Red de Revistas Científicas de América Latina y el Caribe, España y Portugal Sistema de Información Científica Educación, 31 (1). Recuperado de:

[16] Goleman, D. (1996). Inteligencia emocional Kairós: Barcelona.

[17] Hernández, M y Coronado, O. (2007). Desempeño académico de universitarios en relación con ansiedad escolar y autoevaluación. Recuperado de http://repository.ucatolica.edu.co:8080/bits tream/10983/569/1/v11n1a02.pdf

[17] Horna, F. (2001).Aprendiendo a disfrutar del estudio. Lima: remalsa

http://www.redalyc.org/articulo.oa?id=440 31103

[18] Ibarra, L. Educar en la escuela, educar en la familia. ¿Realidad o utopía?, Universidad de Guayaquil, Ecuador, 2002

[19] Loret de mola, J (2011).Estilos y estrategias de aprendizaje en el rendimiento académico de los estudiantes de la universidad peruana "los andes de Huancayo". Revista estilos de aprendizaje n (8). Recuperado de https://es.scribd.com/doc/154884432/TESI S-Estillos-y-Estrategias-en-ElRendimiento-Academico-Lsr-8-articulo-9
[20] Luque Mario (2006) realizó una investigación titulada "Influencia de los hábitos de estudio en el rendimiento académico del área de matemática de los alumnos de la I.E.S de Cabana-2006, Perú

[21] McClellan, D. y Katz, L. (1996). El desarrollo social de los niños: una lista de cotejo. Recuperado de: http: //ericeece.org/pubs/digests/1996/cotej96s.h tml.

[22] Navarro, P. (2006). Prepara oposiciones con éxito: las técnicas y los trucos para superar todas las pruebas. Editorial Planeta Barcelona.

[23] Núñez, C. \& Sánchez, J. (2001). Hábitos de estudio y rendimiento académico. Pontificia Universidad Católica de Chile, Santiago de Chile. Visto el 21 de diciembre del 2011 Recuperado de s.scribd.com/doc/53000062/la-tesisparte2-capitulo-i

[24] Oré, R. (2012).Comprensión lectora, hábitos de estudio y rendimiento académico en estudiantes de primer año de una universidad privada de Lima Metropolitana. (Tesis de maestría) Universidad Nacional Mayor de San Marcos

[25] Ortega, V. (2012).Hábitos de estudio y rendimiento académico en estudiantes de segundo de secundaria de una institución educativa del Callao. (Tesis de maestría) Universidad San Ignacio de Loyola

[26] Pérez, A., Ramón, J., \& Sánchez, J. (2000). Análisis exploratorio de las variables que condicionan el rendimiento académico. Sevilla: Universidad Pablo de Olavide

[27] Pérez, R. (2015). Estilos de aprendizaje y el rendimiento académico en estudiantes de Enfermería de la Universidad Alas Peruanas. Tesis para optar el grado de Magister en Docencia e Investigación en Salud.

[28] Pico, C. (2014). Hábitos de estudio y su incidencia en el rendimiento académico del área de ciencias naturales de los estudiantes de cuarto y quinto año de educación básica de la escuela fiscal mixta Fernando Villacis de la parroquia Sangolqui, Cantón Rumiñahui, provincia de pichincha. Universidad técnica de Ambato. Ecuador

[29] Piñero, L.J.; Rodríguez A. (1998). Los insumos escolares en la educación secundaria y su efecto sobre el rendimiento académico de los estudiantes. Recuperado de 
http://documents.worldbank.org/curated/en /872971468031567258/Los-insumosescolares-en-la-educacion-secundaria-ysu-efecto-sobre-el-rendimiento-academicode-los-estudiantes-un-estudio-en-Colombia

[30] Purihuaman, C. (2013) Estilos de aprendizaje y el rendimiento académico en los estudiantes del I y II ciclo de Ingeniería Civil de la Universidad Cesar Vallejo. Tesis para optar el grado de Magister en Investigación. Universidad Señor de Sipán. Chiclayo, Perú.

[31] Soto, R. (2004). Técnicas de estudio. Lima: Palomino

[32] Sternberg, R.; Detterman, Douglas K. (1992). ¿Qué es la inteligencia?: Enfoque actual de su naturaleza y definición. Madrid: Pirámide, S.A.

[33] Tejedor, F \& García, A (Enero-abril 2007) Causas del bajo rendimiento del estudiante universitario (en opinión de los profesores y alumnos). Propuestas de mejora en el marco del EEES. Revista de Educación, 342., pp. 443-473 Recuperado en http://www.revistaeducacion.mec.es/re342/ re342_21.pdf Fecha de acceso 12/12/2014

[34] Tobón, S (2011) Evaluación de las competencias en la educación básica. (1era edición) México: Santillana Recuperado en http://www.cife.edu.mx/index.php/bibliote ca-digital/evaluacion-de-las-competencias Fecha de acceso 31 de enero, 2015

[35] Vicuña, L. (2005). Inventario de hábitos de estudio. Lima: CEDEIS.

[36] Vildoso, V. (2003). Influencia de hábitos de estudio y la autoestima en el rendimiento académico de los estudiantes de la Escuela Profesional de Agronomía. Universidad Nacional Jorge Basadre Grohmann, Perú. Recuperado de http://www.soarem.org.ar/Documentos/48 $\% 20$ Mena.pdf.

[37] Woolfolk, A. (1995). Psicología Educativa. México: Prentice Hall Hispanoamericana, S.A. 